\title{
Primary Paratesticular Mucinous "Ovarian-Type" Adenocarcinoma: A Rare Case of Scrotal Tumor in a Patient With History of Bilateral Cryptorchidism
}

\author{
Carmelo Agostino Di Franco ${ }^{\mathrm{a}, \mathrm{c}}$, Daniele Porru ${ }^{\mathrm{a}}$, Alessandra Viglio ${ }^{\mathrm{b}}$, \\ Marco Paullib, Bruno Rovereto ${ }^{\mathrm{a}}$
}

\begin{abstract}
Ovarian-type epithelial tumors are extremely rare in testis and paratestis, and in literature no cases of this tumor are reported in a patient with history of bilateral cryptorchidism. We describe a case of an 81-year-old man with a scrotal ulcerative lesion associated with signs of infection. After a skin biopsy showing tumor cells and mucin, we performed a surgical resection of this scrotal lesion and the final pathologic examination revealed a mucinous adenocarcinoma with medium grade of differentiation. The pathologist suggested performing additional tests of this tumor to differentiate it from mucinous "ovarian-type" paratesticular tumor and from a metastatic tumor. We did not find any tumor of different type. We discuss clinical presentation, differential diagnosis and treatment of this rare tumor.
\end{abstract}

Keywords: Paratesticular cancer; Ovarian-type; Mucinous adenocarcinoma; Scrotal mucinous tumor

\section{Introduction}

Surface epithelial tumors are the most common type of neoplasms growing in the ovary and a similar spectrum of tumors can occur in testis and paratestis. Borderline tumors of serous epithelial type are the most frequent subtype within this category, whereas mucinous "ovarian-type" epithelial tumors of testis and paratestis are extremely rare. In literature, no cases of this tumor are described in a patient with a history of cryptorchidism. In this paper, we present a case of primitive mucinous paratesticular "ovarian-type" cancer in an 81-year-old man with a history of bilateral cryptorchidism.

\footnotetext{
Manuscript accepted for publication September 25, 2015

aUrology Department, Fondazione IRCCS Policlinico San Matteo, Pavia, Italy bPathology Unit, Fondazione IRCCS Policlinico San Matteo, Pavia, Italy ${ }^{\mathrm{c} C}$ Corresponding Author: Carmelo Agostino Di Franco, Urology Department, Fondazione IRCCS Policlinico San Matteo, Pavia, Italy.

Email: carmelo_difranco@tiscali.it
}

doi: http://dx.doi.org/10.14740/wjnu235w

\section{Case Report}

We admitted an 81-year-old man to our hospital for fever and a scrotal ulcerative lesion with clear signs of infection. His past medical history included bilateral cryptorchidism surgically treated in young age, inguinal hernia and hypertension. Scrotal examination showed an indolent malodorous scrotal ulcer, discharging abundant purulent and mucinous fluid. We performed a bacterial swab and cold biopsy and started empirical antibiotic therapy. After a few days, fever disappeared and white blood cells dropped within normal range. Bacterial swab was positive for Citrobacter farmeri and Staphylococcus saprophyticus, therefore, we started a targeted antibiotic therapy. Biopsy showed atypical cells, macrophages, bacteria and mucin. Abdomen/pelvis CT scan (Fig. 1) revealed a massive left inguinal hernia containing fat and bowel and a small contralateral inguinal hernia containing only adipose tissue. CT showed a large perineal and scrotal area with multicystic aspects and air microbubbles, as a result of an abscess (Fig. $2,3)$; no intraperitoneal or retroperitoneal fluid was observed.

We decided to perform a surgical resection of the scrotal

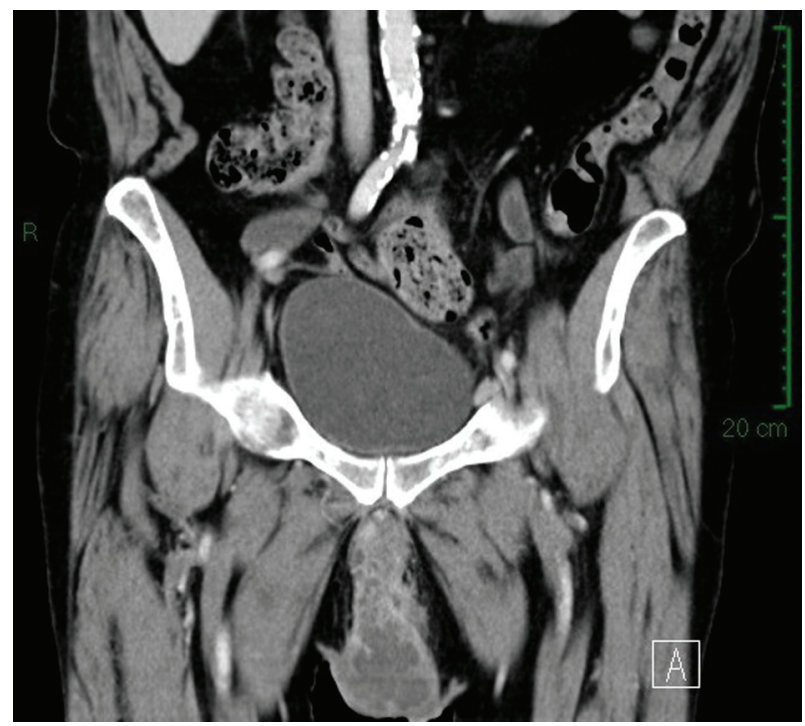

Figure 1. CT scan image: sagittal section of scrotal area showing multicystic aspect of neoplasm. 


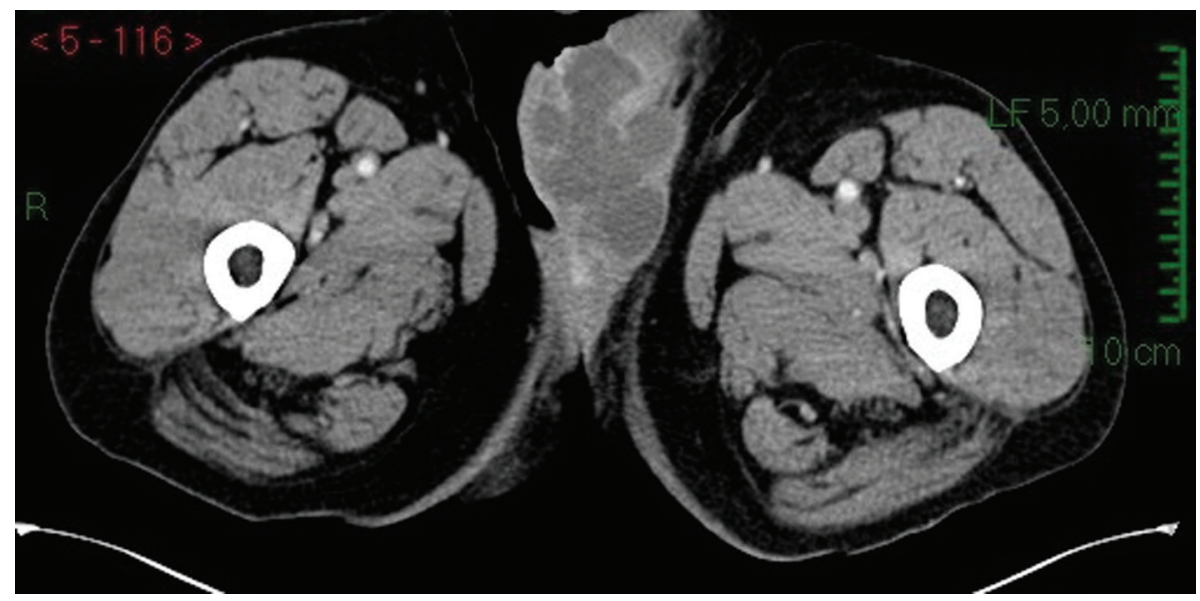

Figure 2. CT scan image: transversal section of scrotal area in arterial phase.

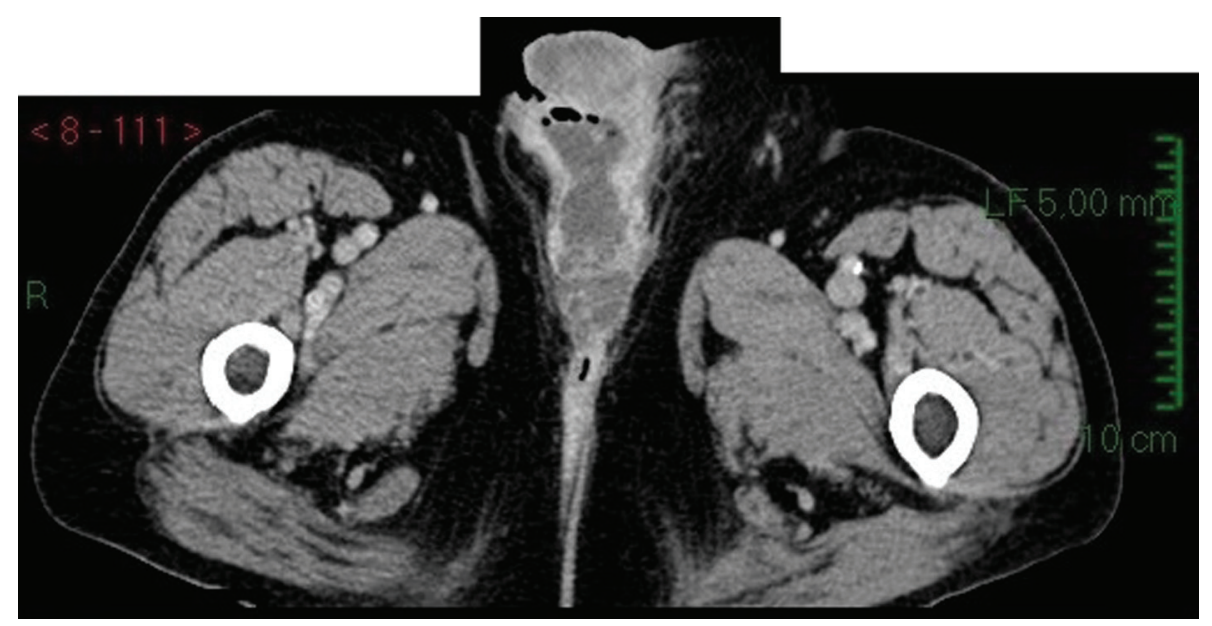

Figure 3. CT scan image: transversal section of scrotal area in venous phase showing contrast enhancement of tumor tissue.

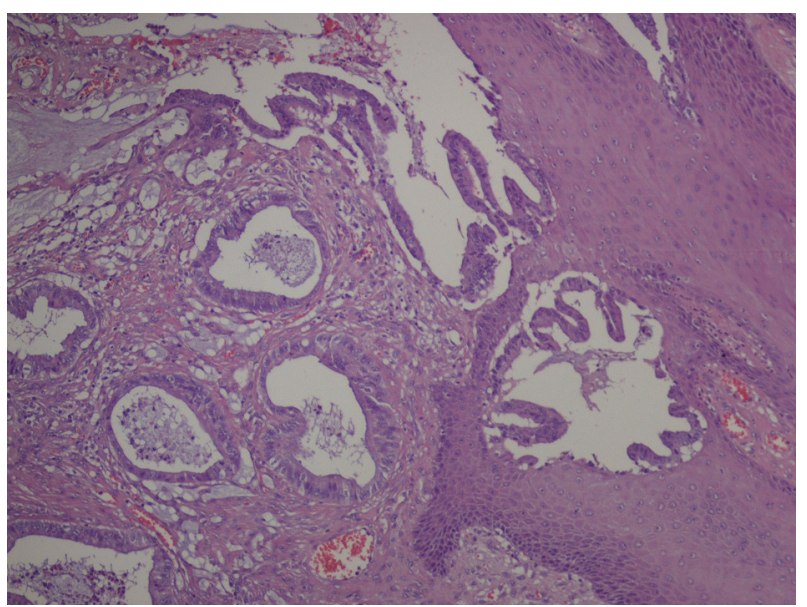

Figure 4. Microscopic image: cystic tumoral tissue with atypical cells and abundant mucin.

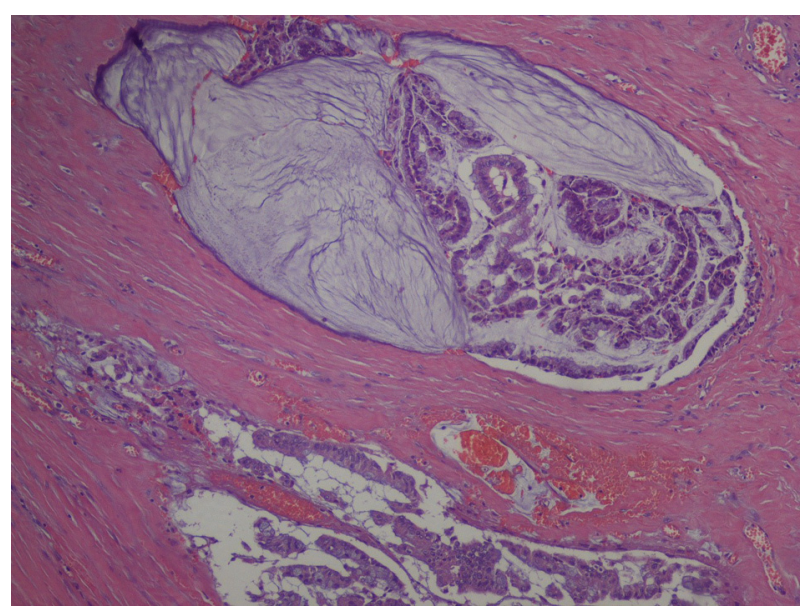

Figure 5. Microscope image (hight magnification): ectasic glandular structures, dysplastic cells with hypercromatic nuclei. 


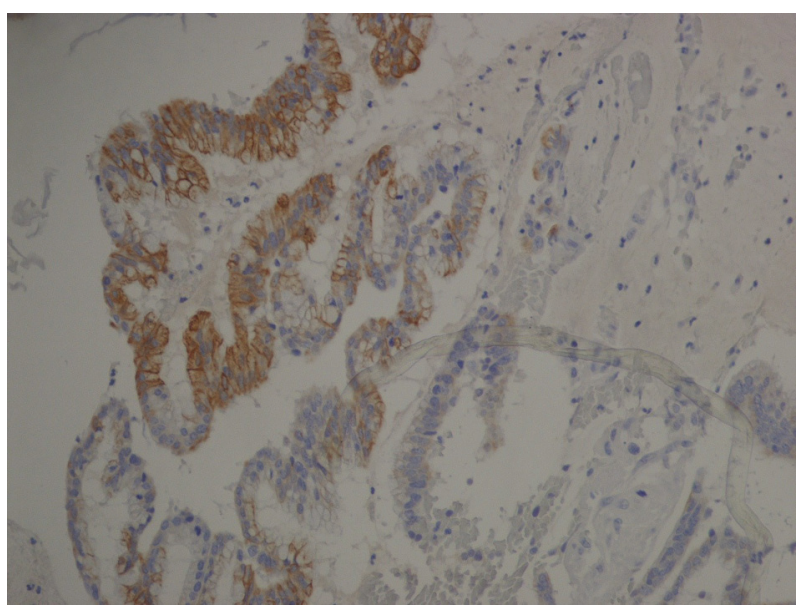

Figure 6. Immunophenotype image: cytocheratine 20+ (brown color). Cytocheratine 20 is expressed by mature enterocytes and goblet cells and is specifically found in the gastric and intestinal mucosa.

mass. We removed a $10 \mathrm{~cm}$ skin flap with underlying soft tissue and epidermal ulcerative lesions. Subcutaneous tissue presented cystic aspects with clear mucinous material. Microscopically (Fig. 4, 5), cysts consist of atypical epithelial columnar cells with mucinous citoplasm, some goblet cells, forming abnormal ectasic glandular structures and abundant extracellular mucus.

Immunophenotype was: cytocheratine $20+, \mathrm{CDX} 2 \mathrm{a}+$, cytocheratine 7-, WT1-, negative estrogen and progesteron receptors; stromal and vascular invasion was observed (Fig. 6, 7).

Histological diagnosis was mucinous adenocarcinoma with medium grade differentiation. Differential diagnosis was between a primitive mucinous paratesticular cancer "ovariantype" and metastasis of gastrointestinal cancer, specifically of cecum or right colon. Pathologist suggested performing further investigation.

We performed serum tumor markers for bowel, lung, pancreatic cancer with negative results; PSA was within range. Colonoscopy revealed a tubulo-villous adenoma of right colon with low-grade dysplasia, no signs of cancerization.

The chest X-ray and chest CT scan had negative results for metastasis. The patient received oncologic follow-up and we started an active surveillance.

\section{Discussion}

Paratesticular tumors are rare, and it is difficult to establish the original organ because tunica vaginalis, epididymis and spermatic cord tissues could potentially turn into cancer. Ovariantype epithelial tumors are very rare in testis and paratestis. In literature, there is very little information about mucinous ovarian-type paratesticular tumors and no case report about this type of cancer in a patient with a history of cryptorchidism. Ulbright and Young [1] reported nine cases of primary mucinous tumor of testis and paratestis with different malignancy grade (benign, borderline and malignant). We now have a follow-up of 3 years of our patient; he had active surveillance without any adjuvant therapy. At present, no metastasis developed.

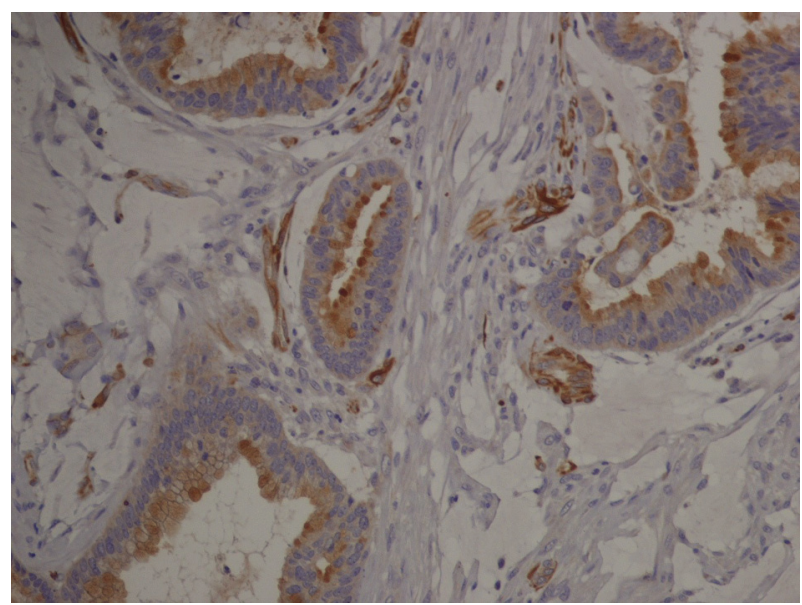

Figure 7. Immunophenotype image (hight magnification): tumor cells expressing cytocheratin 20 are organized in glandular structures and show vascular and stromal invasion.

Mucinous scrotal tumors have a histological pattern similar to ovarian tumors [2], since both show intestinal and endocervical-like features. Their origin could be from Mullerian metaplasia of tunica vaginalis, from Mullerian ducts remnants or from the appendix testis. Our patient has a history of cryptorchidism surgically treated in young age, so we could associate this tumor to the persistent Mullerian duct syndrome, but we have no genetic information about it.

In a patient with this type of cancer, it is necessary to exclude metastasis from other sites. The potential primary sites are prostate, kidney, right colon or cecum [3] or appendix, breast, lung. Seo et al [4] described a paratesticular mucinous metastasis from pancreatic cancer, and in literature, less than 20 metastatic cases have been reported.

In our patient, tumor markers and PSA were negative, digital rectal examination was normal, thoraco-abdominal CT and colonoscopy were negative for cancer. We observed that the tumor has a high infectious risk due to its mucinous content, and our patient had citrobacter and staphylococcus infection, which if not identified and treated could evolve in serious complications.

\section{Conclusion}

Primary paratesticular ovarian-type mucinous adenocarcinoma is a very rare tumor of testis and paratestis. Usually, this cancer has a benign or borderline behaviour, with a low risk of progression and metastases. The most important step is to define correctly the nature of tumor with a differential diagnosis, to exclude an aggressive clinical behaviour, treat possible associated infections and establish a targeted oncologic follow-up.

\section{References}

1. Ulbright TM, Young RH. Primary mucinous tumors of the testis and paratestis: a report of nine cases. Am J Surg 
Pathol. 2003;27(9):1221-1228

2. Menon S, Ahmed S, Desai S. Primary borderline mucinous neoplasm of the testis: A case report and literature review. Indian J Urol. 2012;28(2):224-226.

3. Tanaka H, Yasui T, Watase H. [Metastatic tumor of the epididymis from pancreatic carcinoma: a case report]. Hinyokika Kiyo. 1999;45(9):649-652.

4. Seo IY, Kim SG, Han WC, Rim JS. Paratesticular mucinous cystadenocarcinoma: metastasis from pancreatic cancer. Int J Urol. 2004;11(12):1147-1149. 